\title{
Phonon-assisted carrier transport through a lattice-mismatched interface
}

\author{
Hyong Seo Yoon ${ }^{1}$, Juyeong Oh ${ }^{1}$, Jae Young Park', JeongSeob Kang ${ }^{1}$, Junyoung Kwon², Teresa Cusati ${ }^{3}$, Gianluca Fiori ${ }^{3}$, \\ Giuseppe lannaccone ${ }^{3}$, Alessandro Fortunelli $\mathbb{C}^{4}$, V. Ongun Ozcelik $\mathbb{B}^{5}$, Gwan-Hyoung Lee $\mathbb{B}^{2,6}$, Tony Low ${ }^{7}$ and \\ Seong Chan Jun ${ }^{1}$
}

\begin{abstract}
$\mathrm{MoS}_{2}$ typically exhibits unconventional layer-thickness-dependent electronic properties. It also exhibits layerdependent band structures including indirect-to-direct band transitions, owing to which the electronic and carrier transport properties of a lattice-mismatched, conducting, two-dimensional junction are distinct with the naturally stepwise junction behaving as a 1D junction. We found distinguishable effects at the interface of vertically stacked $\mathrm{MoS}_{2}$. The results revealed that misorientationally stacked layers exhibited significantly low junction resistance and independent energy bandgaps without bending owing to their effectively decoupled behavior. Further, phononassisted carriers dominantly affected the lattice-mismatched interface owing to its low junction resistance, as determined via low-temperature measurement. Our results could facilitate the realization of high-performance $\mathrm{MoS}_{2}$ transistors with small contact resistances caused by lattice mismatching.
\end{abstract}

\section{Introduction}

Two-dimensional (2D) semiconductor materials have recently received extensive attention because of their large bandgap ${ }^{1-10}$. Among 2D semiconductors, transition-metal dichalcogenides (TMDs), specifically $\mathrm{MoS}_{2}$, are considered as promising materials for advanced electronic devices based on their stable electronic properties and high feasibility for large-scale mass production ${ }^{11-24}$. Furthermore, their electrical properties depend on their atomicstructure deformation ${ }^{13}$ and surface defect concentration $^{14}$, and they can be tuned over a wide range by changing their thickness ${ }^{12}$. For example, $\mathrm{MoS}_{2}$ transistors generally exhibit $\mathrm{n}$-type conduction in a standard transistor structure. However, ambipolar behavior can be engineered via dielectric and substrate effects, and even p-type

\footnotetext{
Correspondence: Gwan-Hyoung Lee (gwanlee@yonsei.ac.kr) or Seong Chan Jun (scj@yonsei.ac.kr)

${ }^{1}$ School of Mechanical Engineering, Yonsei University, Seoul 03722, Korea

${ }^{2}$ Department of Materials Science and Engineering, Yonsei University, Seoul 03722, Korea

Full list of author information is available at the end of the article.

These authors contributed equally to this work: Hyong Seo Yoon, Juyeong Oh, Jae Young Park
}

characteristics were obtained by applying specific metaloxide contacts ${ }^{15,16,25}$. In addition, an anomalous bandstructure transition from indirect to direct was observed while thinning $\mathrm{MoS}_{2}$ to a single atomic layer ${ }^{26,27}$.

To utilize 2D TMDs as a homojunction device, a difference in thickness is necessary. However, homojunctions using 2D TMDs have a perfectly aligned stepwise junction, which is distinct from a randomly stacked homojunction. Thus far, there have not been systematic studies on the electronic properties of these two types of junctions. We fabricated field effect transistor (FET) devices with a single $\mathrm{MoS}_{2}$ flake that consisted of different numbers of layers ranging from thin (less than three layers) to thick (more than four layers). Such devices are termed "naturally stepwise junction" (NSJ) devices. Subsequently, we compared the performance of the NSJ devices with that of a lattice-mismatched junction (LMJ) device. Although both type-I and type-II junctions have been reported recently for the NSJ structure ${ }^{28,29}$, our experimental and calculational results are consistent with a type-I junction. In this study, surface-potential measurements using the scanning Kelvin probe microscopy (SKPM) technique allowed us to

\section{(c) The Author(s) 2019}

(c) (i) Open Access This article is licensed under a Creative Commons Attribution 4.0 International License, which permits use, sharing, adaptation, distribution and reproduction cc) in any medium or format, as long as you give appropriate credit to the original author(s) and the source, provide a link to the Creative Commons license, and indicate if changes were made. The images or other third party material in this article are included in the article's Creative Commons license, unless indicated otherwise in a credit line to the material. If material is not included in the article's Creative Commons license and your intended use is not permitted by statutory regulation or exceeds the permitted use, you will need to obtain permission directly from the copyright holder. To view a copy of this license, visit http://creativecommons.org/licenses/by/4.0/. 
determine the work function of $\mathrm{MoS}_{2}$ for different layer thicknesses. In addition, we could deduce the junction type from the conduction-band minimum (CBM) and valence band maximum (VBM) obtained from density functional theory (DFT). For the LMJ structure, the potential barrier was very small, and layer decoupling at the interface was observed. Furthermore, the band structures of LMJ and NSJ as well as temperature-dependent current values were investigated.

\section{Materials and methods \\ Device fabrication}

Figure 1a shows a schematic of the fabricated device. The $\mathrm{MoS}_{2}$ devices had a thickness step in the middle, which was formed via two different approaches. For the ohmic contact, graphene electrodes were adopted and deposited $\mathrm{Pd} / \mathrm{Cr} / \mathrm{Au}(30 \mathrm{~nm} / 5 \mathrm{~nm} / 20 \mathrm{~nm})$ as metal pads (Fig. S2). Metal electrodes were patterned by e-beam lithography (TESCAN VEGA3). For the NSJ, we used a $\mathrm{MoS}_{2}$ flake including naturally adjacent atomic steps with a perfectly aligned atomic lattice (Fig. 1b). The LMJ-MoS devices were produced by transferring a thicker $\mathrm{MoS}_{2}$ flake onto a thinner one using a transferring device (MSTech Probe Station Series MST 5500B, Fig. S1).

\section{Characterization of junction devices}

To compare transport characteristics between NSJ and LMJ, both types of devices were experimentally measured and theoretically analyzed via surface potential measurement, I-V measurement with temperature variation, $\mathrm{Y}-$ function analysis, and DFT analysis. The surface potential value was obtained using SKPM, and the work function translated from the value was obtained through Eq. (1) in chapter 2 of the supporting information. CBM and VBM were obtained by applying DFT, which is the theoretical calculation method (see chapter 4 of the supporting information). For the geometry optimization calculations, the Brillouin zone (BZ) was sampled in the MonkhorstPack scheme where the convergence of energy as a function of the number of $\mathbf{k}$-points was tested. The Yfunction method was used to obtain the contact resistance. The data obtained from the I-V results were calculated using Eqs. (2-5) from the supporting information for strong inversion cases. Additionally, using Eq. (6) from the supporting information to plot Arrhenius, we deduced the junction barrier height. In this work, each I-V data was measured in a low-temperature chamber and controller (LakeShore 336 Temperature controller) since we had to know the change in temperature.

\section{Results and discussion}

Layer dependent homojunction of $\mathrm{MoS}_{2}$

Figure $1 \mathrm{~d}$ shows the output curves of a representative NSJ-MoS ${ }_{2}$ device consisting of two and five layers clearly indicating the quasi-current-rectifying effect and the ntype response to the back-gate voltage. Such asymmetrical current-voltage behavior implies a built-in electric field at the junction, which can arise from the thicknessdependent electron affinity and bandgap of $\mathrm{MoS}_{2}{ }^{12,26,27,30-33}$. The LMJ-MoS 2 device, fabricated by transferring a six-layer (6L) $\mathrm{MoS}_{2}$ onto a $2 \mathrm{~L}$ flake yielding a large-area 2D interface, as shown in Fig. 1c, and the corresponding output curves are presented in Fig. 1e. Contrary to the NSJ-MoS $\mathrm{Mevice}_{2}$ these output curves are linear and symmetric indicating that the misorientationally stacked device had ohmic-like contacts at the vdW interface (rectification ratio for both types can be seen in Fig. S3).

\section{Naturally stepwise junction (NSJ)}

To understand the band alignment of the layerdependent $\mathrm{MoS}_{2}$ junction, we evaluated the layerdependent work function using SKPM (Fig. S4). Figure 2a shows the evolution of the work function, CBM, and VBM of $\mathrm{MoS}_{2}$ with layer number. The work function is obtained via SKPM, while CBM and VBM values are from the DFT calculation. The surface potential of the $\mathrm{MoS}_{2}$ layer decreases with layer number indicating an increase in the work function (Figs. S5-6). The work function of $\mathrm{MoS}_{2}$ linearly increases for up to five layers and asymptotically approaches $4.68 \mathrm{eV}$. The work function of monolayer $\mathrm{MoS}_{2}$ was $4.43 \mathrm{eV}$, which is similar to the previously reported value ${ }^{34}$. The CBM decreases as the number of layers increases, while the VBM decreases. The CBM of thin $\mathrm{MoS}_{2}$ is higher than that of thick $\mathrm{MoS}_{2}$ and the VBM is lower. Therefore, when two $\mathrm{MoS}_{2}$ that have different thicknesses meet, a type-I junction is formed.

The distinctive difference between the NSJ and LMJ is the lattice alignment. For the LMJ, the lattice structures of the two layers are misoriented with respect to each other. Conversely, in the NSJ, each layer of $\mathrm{MoS}_{2}$ is wellstacked to form smoothly oriented lattice structures. We performed DFT calculations to elucidate the different electronic properties of these two cases (Figs. S10-11). For the NSJ, the interface is considered 1D and the effect of the imperfectly terminated edge of the thicker side can be very significant. Figure $2 \mathrm{c}$ shows the calculated local density of states (LDOS) for the 2L-6L NSJ. Here, the LDOS away from the interface recovers the expected bulk band edges for $2 \mathrm{~L}$ and $6 \mathrm{~L} \mathrm{MoS}_{2}$. However, at the interface, we observed a reduced LDOS at the band edges. This reduced LDOS, albeit only occurring across a few atomic layers, can be a significant source of carrier scattering. By considering the CBM and the work function of each side of the $\mathrm{MoS}_{2}$, the band alignment is determined for the NSJ, as shown in Fig. 2b. The current rectifying behavior is mainly due to the existence of band 
(a)

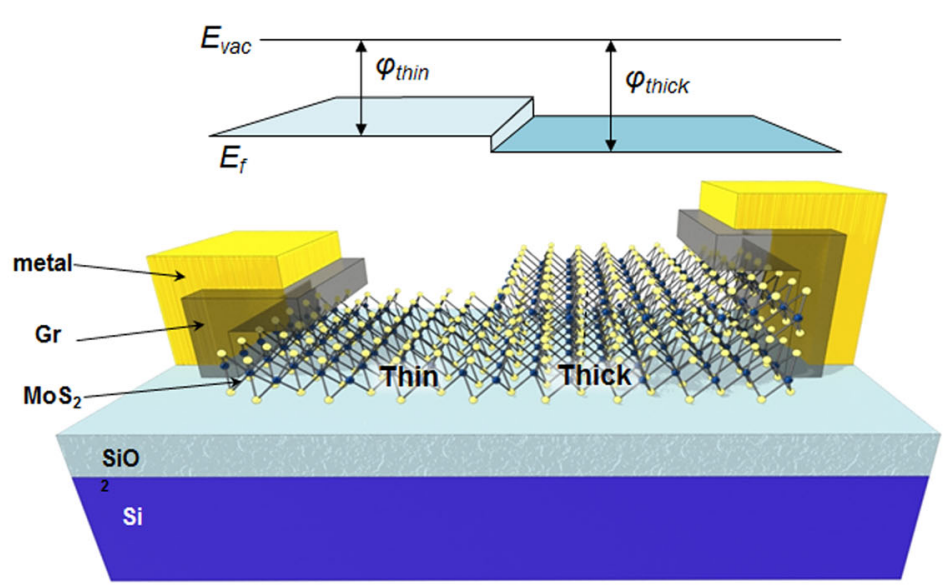

(b)

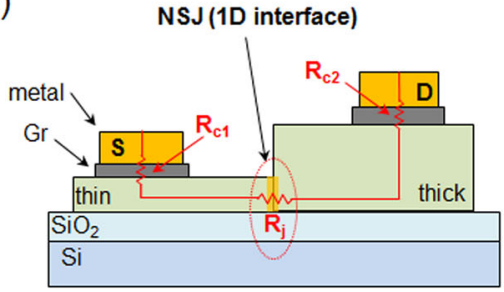

(d)

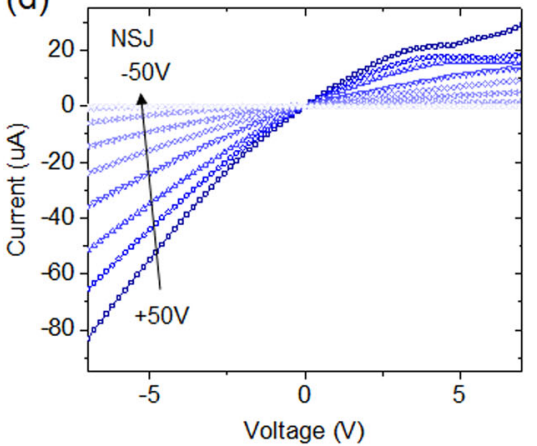

(c)

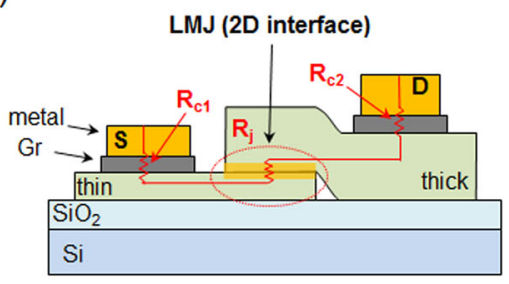

(e)

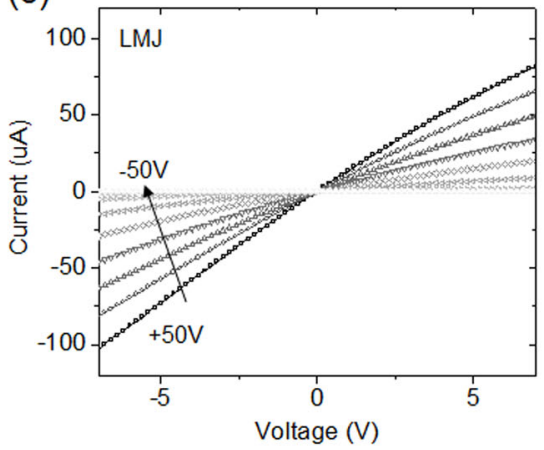

Fig. 1 Images of a FET device with thickness-dependent $\mathrm{MoS}_{2}$ isotype heterojunction with graphene electrodes and Pd metal pads. Our surfacepotential measurement suggests that work-function value increases with thickness of MoS $_{2}$ layer. Schematics for $\mathbf{b}$ NSJ device and $\mathbf{c}$ LMJ device indicating the presence of junction resistance in addition to each contact resistance for layers 1 and 2. Current-voltage relationship at various back gate voltages for $\mathbf{d}$ NSJ and e LMJ

bending and a potential barrier at the interface. We observed a higher current amplitude under negative voltage bias on the thicker layer. Although $\mathrm{MoS}_{2}$ homojunction certainly forms a type I junction, the CBM difference between layers can be much smaller than that of the work function, especially in the case that a monolayer $\mathrm{MoS}_{2}$ is not involved. Therefore, as described in Fig. $2 \mathrm{~b}$, the potential barrier for electrons can be minimized when it comes from a thicker layer to a thinner layer.

\section{Lattice-mismatched junction (LMJ)}

Figure 3a, b show the distribution of the wave function in real space along the layers of a perfectly aligned, uniform 6L $\mathrm{MoS}_{2}$ structure and a misaligned 2L/4L structure, respectively. The results show that the wave functions are well separated between the misaligned $2 \mathrm{~L}$ and $4 \mathrm{~L} \mathrm{MoS}_{2}$ in the latter structure, while the perfectly aligned $6 \mathrm{~L} \mathrm{MoS}_{2}$ has a strongly mixed wave function. Thus, when the lattice structures of randomly stacked $\mathrm{MoS}_{2}$ layers are mismatched, each stacked layer is electronically decoupled and should be regarded as two separate materials. Consequently, the lattice-mismatched homojunction interface is $2 \mathrm{D}$.

Figure 3c presents a surface-potential image of a $\mathrm{MoS}_{2}$ flake obtained by SKPM. The $\mathrm{MoS}_{2}$ flake contains various regions with different thicknesses. In Fig. 3c, we observe a clear transition in the surface-potential values as the 
(a)

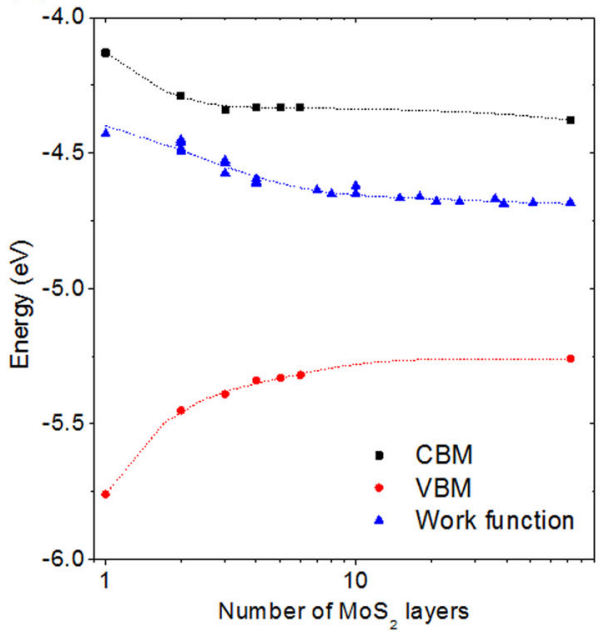

(b)

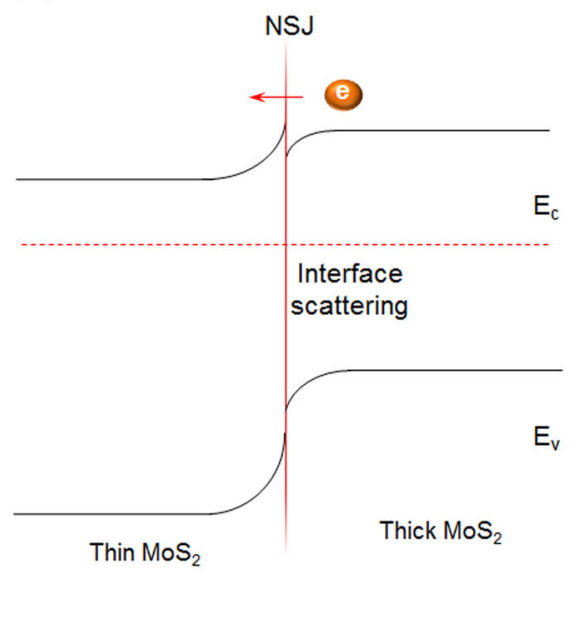

(c)

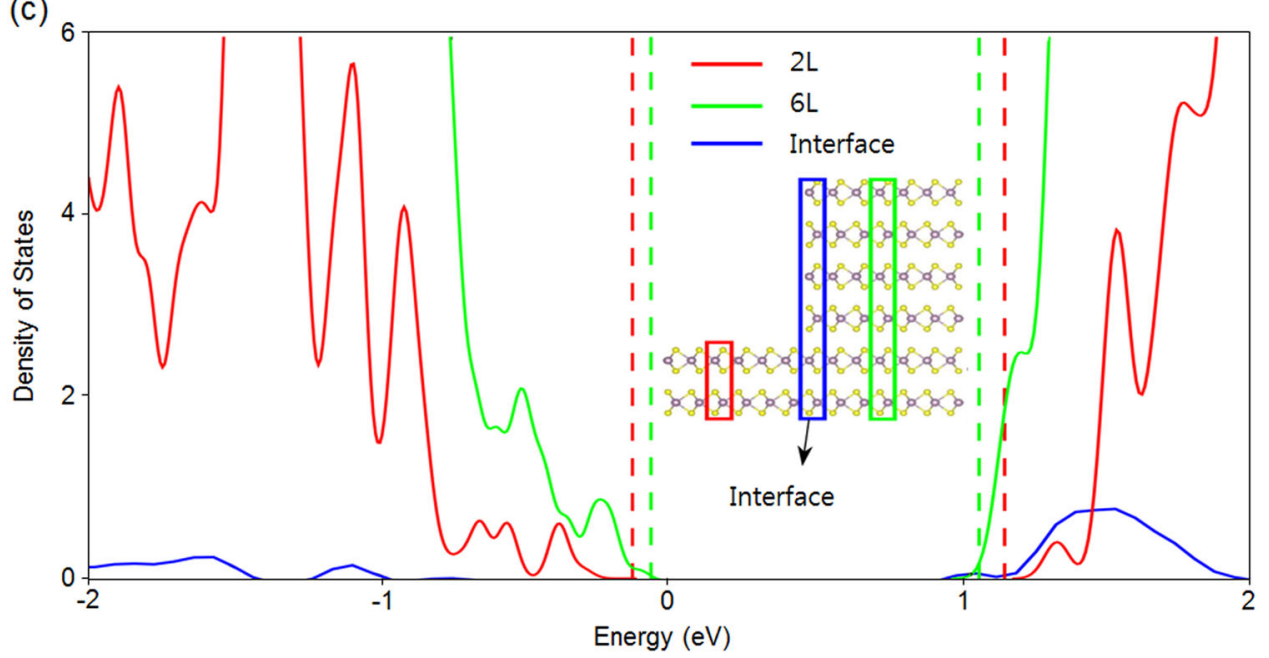

Fig. 2 a Summary of energy band structure of $\mathrm{MoS}_{2}$ with its layer number. Work function values were obtained via SKPM. CBM and VBM values were calculated. The results indicate a consistent increase in work function with layer thickness. $\mathbf{b}$ Energy-band alignment of layer-dependent MoS 2 -NSJ. c Side view of NSJ comprising $6 \mathrm{~L}$ and $2 \mathrm{~L} \mathrm{MoS}_{2}$ structures (inset). LDOS of atoms is indicated in atomic structure. Vertical dashed lines show CBM and VBM energies of uniform $2 \mathrm{~L}$ and $6 \mathrm{~L}$ structures

number of layers is increased from two to seven. As the thickness is increased, the $\mathrm{MoS}_{2}$ nanosheet tends to have a lower surface potential and higher work function. Figure $3 \mathrm{~d}$ shows a surface-potential image of artificially stacked $\mathrm{MoS}_{2}$ flakes, which were fabricated by transferring a $4 \mathrm{~L} \mathrm{MoS}_{2}$ (red dotted line) onto a $2 \mathrm{~L} \mathrm{MoS}_{2}$ (yellow dotted line). There are four separate regions: $2 \mathrm{~L}, 3 \mathrm{~L}$ of the bottom $\mathrm{MoS}_{2}$, $4 \mathrm{~L}$ of the transferred $\mathrm{MoS}_{2}$, and $6 \mathrm{~L}$ of the misorientationlly stacked $\mathrm{MoS}_{2}$. It was confirmed that the 2L, 3L of the bottom $\mathrm{MoS}_{2}$, and 4L of the top $\mathrm{MoS}_{2}$ had distinct surface-potential differences. However, the overlapped region of $6 \mathrm{~L}$ exhibited no surface-potential change compared with the $4 \mathrm{~L}$ of the top $\mathrm{MoS}_{2}$. Figure 3e, f show the results for each sample. While the surface potential of each $\mathrm{MoS}_{2}$ layer exhibits a consistent decrease with layer number, the overlapped layer shows only identical values. This result strongly supports the physical picture of interlayer decoupling owing to lattice-mismatches between the overlapped $\mathrm{MoS}_{2}$ flakes. To systematically analyze these layer-dependent work-function differences and the layer decoupling effect, we extracted the junction resistance of each NSJ and LMJ device.

For an LMJ, the thickness of the device is far smaller than the relevant carrier screening length. Figure $3 g$ shows a rotated schematic of an LMJ device and an energy band diagram for the LMJ case. Since the actual band structure is restricted in atomic thickness, the potential barrier between the two layers can be identified by the CBM difference, and it is much smaller than that of the NSJ case in most cases. Additionally, the LDOS for each atomic region 


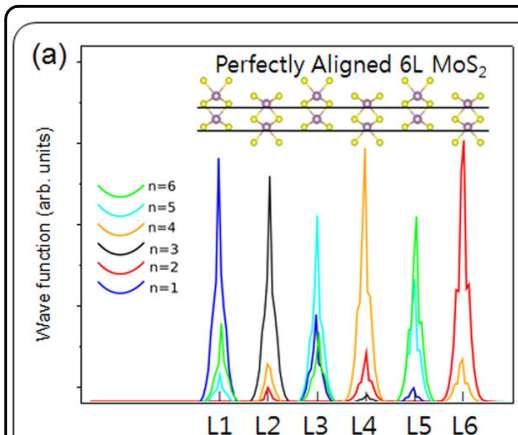

(c)

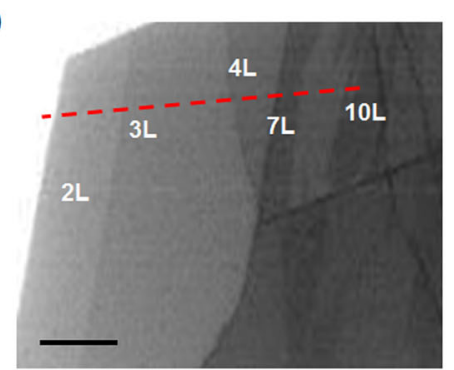

(e)

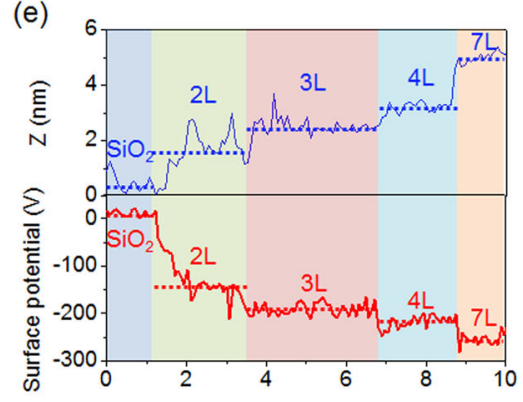

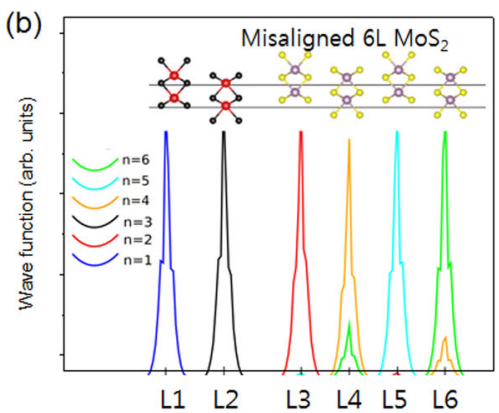

(d)

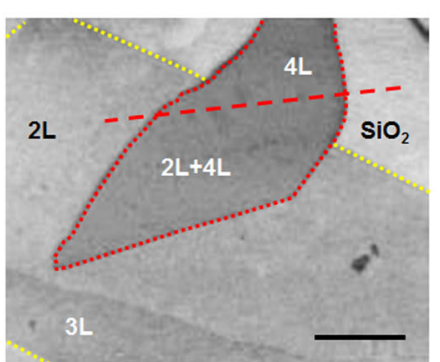

(f)

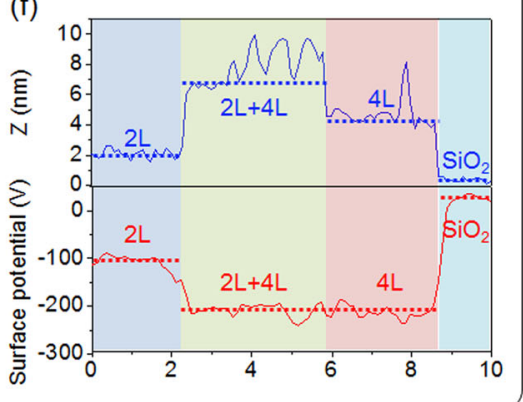

(g) $\mathrm{Si} / \mathrm{SiO}_{2}$

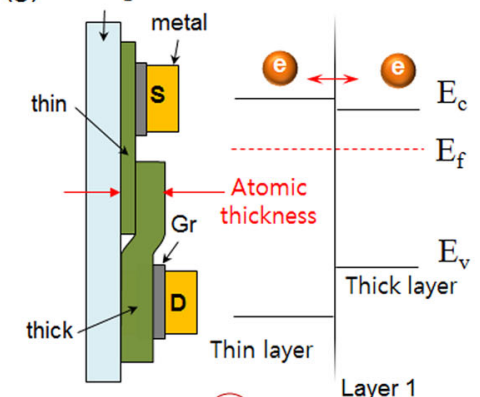

(h)

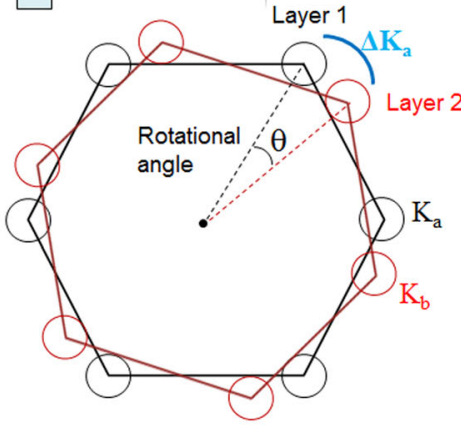

(i) $2.5 .-$ AB stacking $(\mathrm{d}=2.98 \AA \mathrm{A})$

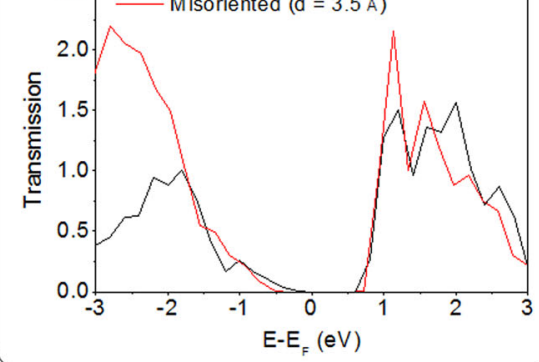

Fig. 3 a Distribution of wave functions in real space along layers of perfectly aligned uniform $6 \mathrm{~L} \mathrm{MoS}_{2}$. The wave functions for each conduction band at point $K$ are represented by different colors, as indicated in the inset, where $n=1$ corresponds to CBM. $\mathbf{b}$ Same as a but for misaligned $6 \mathrm{~L}$ MoS 2 . Here, $2 \mathrm{~L} \mathrm{MoS}$ (indicated by black and red spheres) is shifted and misaligned with respect to the remaining four layers ( $4 \mathrm{~L}$ ) to create the $2 \mathrm{~L}-4 \mathrm{~L}$ interface. Conduction-band wave functions are mixed along layers in $\mathbf{a}$, whereas in $\mathbf{b}$ they are separated from each other at the $2 \mathrm{~L}-4 \mathrm{~L}$ interface. Surface-potential image of $\mathbf{c}$ single $\mathrm{MoS}_{2}$ flake comprising two to 10 layers and $\mathbf{d} \mathrm{MoS}_{2}$ flake comprising two and three layers covered by transferred four-layer $\mathrm{MoS}_{2}$. Measured thickness and potential values corresponding to e single flake and $\mathbf{f}$ covered flake. Covered region (six layer) exhibited identical values to original flake (four layer). $\mathbf{g}$ Schematic of rotated LMJ and energy-band alignment of layer-dependent MoS 2 LMJ. $\mathbf{h}$ Misalignment of misorientationally stacked $\mathrm{MoS}_{2}$ layers in reciprocal space. $\mathbf{i}$ Transmission coefficient as a function of energy computed for the various interlayer conditions

at the interface of LMJ indicated CBM alignment and no significant band shift (Figs. S10-11). In addition, for an LMJ, the relative misorientation of each layer implies a mismatch in reciprocal space, as illustrated in Fig. 3h. Hence, the carrier transport across this 2D interface would require the assistance of a large-momenta supplied by optical phonons. To understand the effect of the lattice misorientation, explicit conductance simulations were performed $^{35}$. The corresponding transmission coefficients are plotted in Fig. 3i. The result of this calculation is that the misoriented junction exhibits similar interlayer conductance with respect to bulk $\mathrm{MoS}_{2}$ and even higher conductance in the valence band region and around CBM. The reasons for such unexpected behavior lie in a "charge compression" effect. In the bulk system, black curve in Fig. 3i, the repulsion of the electronic clouds of the $\mathrm{S}$ atoms pushes the valence band to higher energy, especially at the $\Gamma$ point, producing a decrease in the indirect band gap $^{29,36,37}$. Additionally, the rotational angle in the LMJ case may be another issue, but studies regarding twisted, misaligned TMD heterojunctions and graphene layers suggest an insensitivity to angle ${ }^{38,39}$. Basically, the twist angle controls the effective interlayer coupling and induces a van Hove singularity at some energy ${ }^{39}$. 
Junction resistance analysis by using Y-function method

To quantitatively evaluate the junction resistances at the interfaces, we employed the Y-function method. Figure 4a shows a representative transfer curve of a $\mathrm{MoS}_{2}$ homojunction device. We choose the samples showing gate independent attenuation as "strong inversion" (Fig. S7). Because of the interfaces in both junction devices, the junction resistances should be considered as both effective mobility attenuation factors and the contact resistances. Figure $4 \mathrm{~b}$ shows the extracted sum of the contact resistance and junction resistance as a function of the work function and CBM difference for both homojunction devices. Because the contact resistances are expected to have small values (less than a few $\mathrm{k} \Omega$ ) and do not differ regardless of the device structure and thickness, the difference in the resistance originates entirely from the junction resistance at the interface. The resistance of the NSJ increases exponentially with the surface-potential difference between the two $\mathrm{MoS}_{2}$ layers. It is evident that the barrier resulting from the band bending in the NSJ case can be determined by the Fermi-level difference. This indicates that the junction resistance dominates the total resistance. It also justifies our assumption in the modification of the Y-function method. In the LMJ case, the barrier at the interface can be determined by the alignment of the CBM in equilibrium. The suggested values in Fig. $4 \mathrm{~b}$ are given by the work function and CBM transition shown in Fig. 2a. The LMJ devices exhibit significantly smaller resistances than the NSJ devices in most cases and show a rough dependence on the energy difference, although the LMJ cases have many uncontrolled variables at the interface (Figs. S8-9). We measured the entire devices at room temperature, and the phonon-assist effect may have been significant. Cases with an energy difference smaller than $50 \mathrm{meV}$ exhibit particularly small resistance values. Furthermore, at energies higher than $50 \mathrm{meV}$, the resistance is drastically increased to values comparable to the NSJ cases. The phonon-assisted charge-transfer mechanism may explain this behavior given the reported optical phonon energy of $\mathrm{MoS}_{2}{ }^{40}$.

To further verify this conclusion, we fabricated both types of $\mathrm{MoS}_{2}$ homojunction devices on the same $\mathrm{MoS}_{2}$
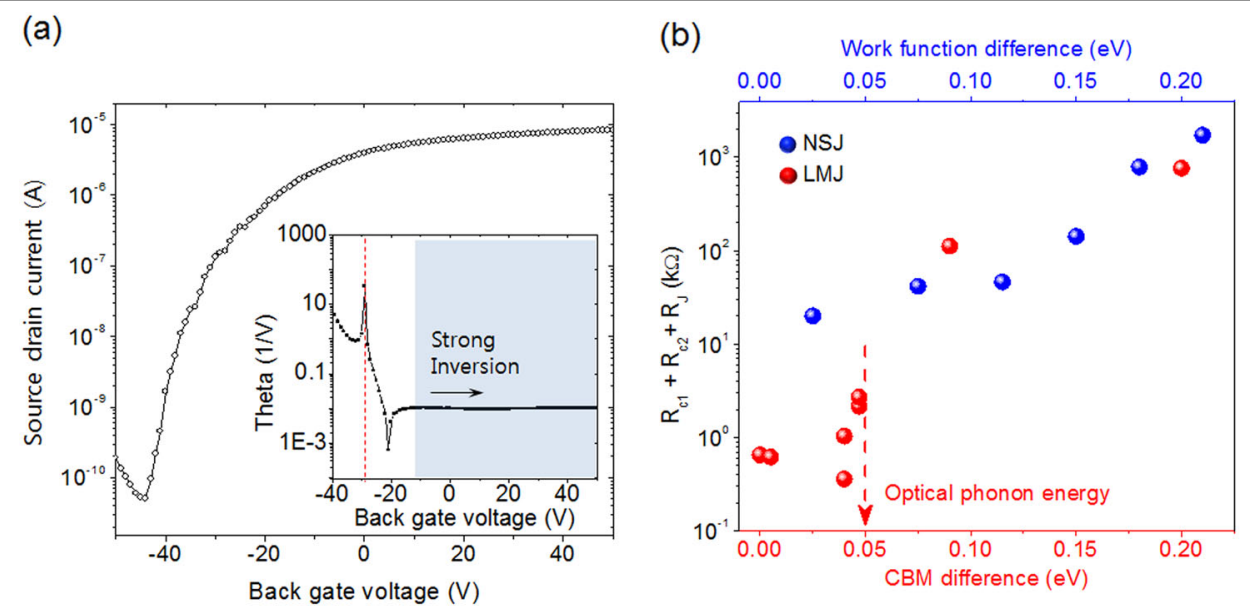

(c)

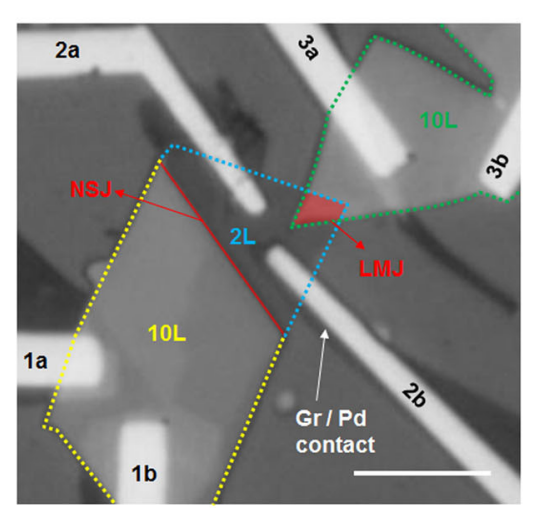

(d)

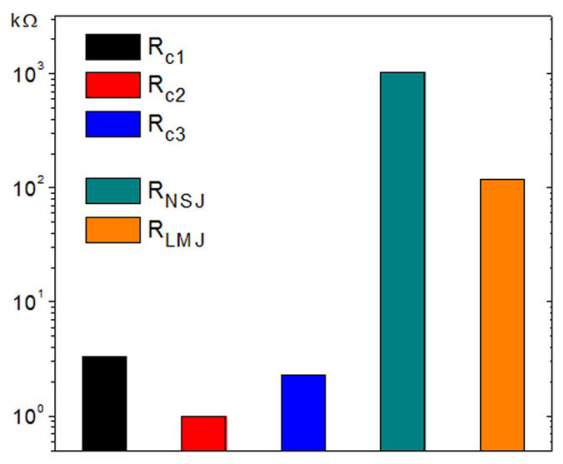

Fig. 4 a The transfer curve of $\mathrm{MoS}_{2}$ homojunction transistor (inset). The mobility attenuation factor, $\theta$, with back gate bias. b Sum of contact resistance and junction resistance for various homojunction devices extracted using Y-function analysis. c Device image designed to separate junction resistance from contact resistance. Contact resistance was determined by measuring a pair of electrodes on the same $\mathrm{MoS}_{2}$ layer $(1 \mathrm{a}-1 \mathrm{~b}$, 2a-2b, and 3a-3b). d Extracted junction resistance for NSJ and LMJ cases 
flake, as shown in Fig. 4c. We used $\mathrm{MoS}_{2}$ flakes with $2 \mathrm{~L}$ (blue dotted line) and 10L (yellow dotted line) regions for the NSJ. Another $\mathrm{MoS}_{2}$ flake of 10L was transferred onto the $2 \mathrm{~L}$ region for forming the LMJ. The $\mathrm{MoS}_{2}$ devices were measured by using $1 a-1 b, 2 a-2 b$, and $3 a-3 b$ contacts where the contact resistances for the three devices were extracted via the Y-function method. The contact resistances for the three devices were significantly smaller than the total device resistance $(<5 \mathrm{k} \Omega)$ (Fig. $4 \mathrm{~d})$. Both devices were measured by using $1 \mathrm{a}-2 \mathrm{a}$ and $2 \mathrm{a}-3 \mathrm{a}$ contact cases. By substituting the measured contact resistance from the total resistance, we separated the junction resistance for each device. The junction resistance of the LMJ device was significantly smaller than that of the NSJ device. For all NSJ cases and most LMJ cases, it is reasonable to assume that the contact resistance has a negligible contribution to the total device resistance. Thus, we conclude that the carrier transport mechanisms in the both $\mathrm{MoS}_{2}$ homojunction cases differ significantly. While the NSJ case exhibits a strong potential-barrier dependence based on the work-function difference, the decoupled LMJ emphasizes the importance of phonons in the charge transfer across the misaligned lattices.

\section{Junction barrier height characterization of homojunctions by temperature modulation}

In intrinsic $\mathrm{MoS}_{2}$ devices, the temperature affects the $\mathrm{I}-\mathrm{V}$ characteristics in two dominant ways. The first is the thermionic effect where the number of charge carriers increases as the temperature rises from low to high. In contrast, phonon scattering, which interferes with the movement of electrons, increases as the temperature rises. In junction devices, there is another way for temperature to affect the I-V characteristics. After the temperature rises and the phonon scattering energy becomes higher than the barrier height of the junction, the charge carrier can overcome the junction barrier easily by the phononassist effect. Therefore, temperature-dependent electrical measurements were carried out to characterize the specific junction barrier height according to the lattice matching of the junction.

In LMJ, decoupling between two different $\mathrm{MoS}_{2}$ occur causing band bending by Fermi level pinning at the junction to be drastically reduced compared to NSJ (Fig. 5a, b). To observe a reduced barrier height by decreasing Fermi level pinning, two probe based I-V were measured from 77.4 to $300 \mathrm{~K}$. In the case of the NSJ (a)

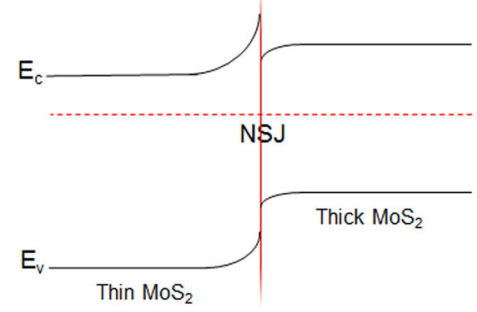

(d)

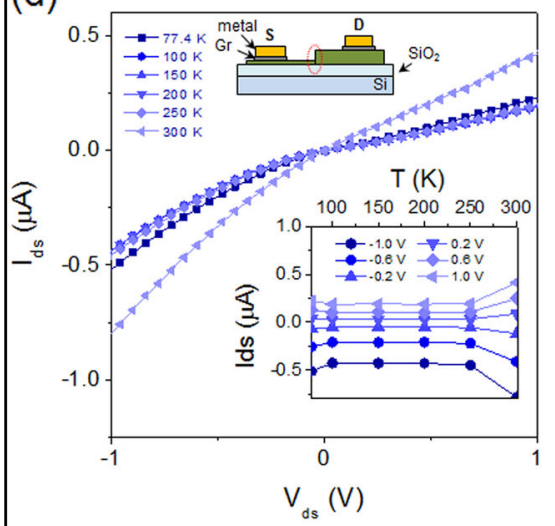

(b)

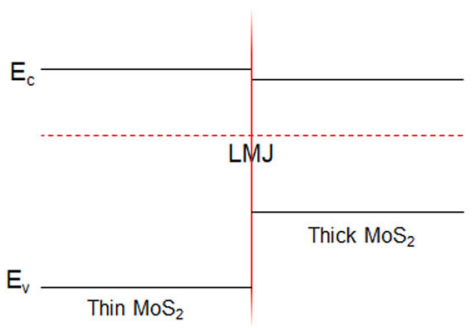

(e)

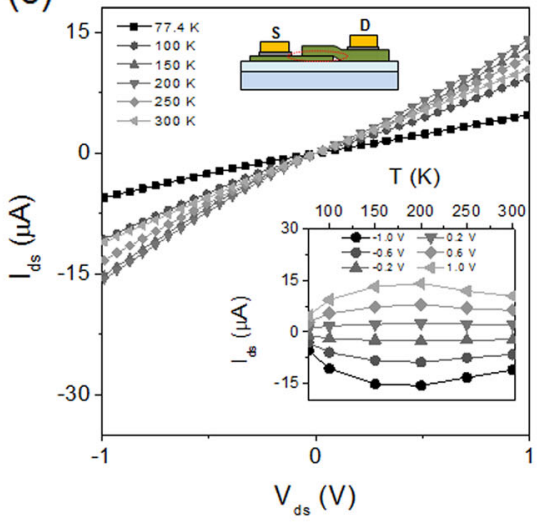

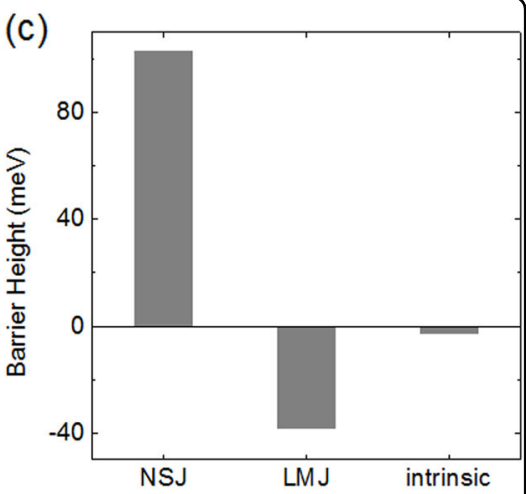

(f)

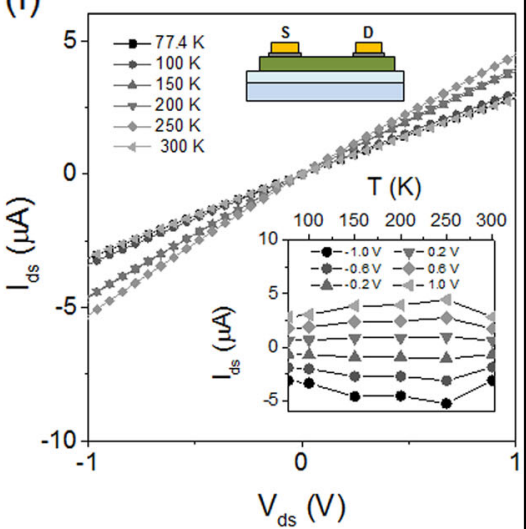

Fig. 5 Energy-band diagrams for $\mathbf{M o S}_{\mathbf{2}}$ a NSJ, and $\mathbf{b}$ LMJ. c The junction barrier height for NSJ, LMJ, and intrinsic MoS 2 . The junction barrier height at the NSJ interface is higher than the junction barrier height at the LMJ interface. The drain current-voltage curves with various temperatures from 77.4 to $300 \mathrm{~K}$ for $\mathbf{d}$ NSJ device, e LMJ device, and $\mathbf{f}$ intrinsic MoS $_{2}$ device, (inset) drain current versus temperature at various drain-source biases 
(a)

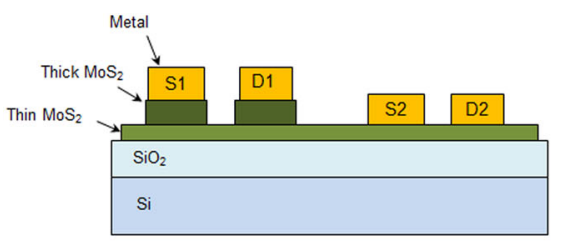

(b)

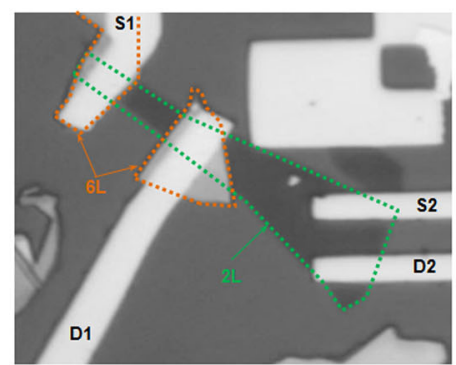

(e)

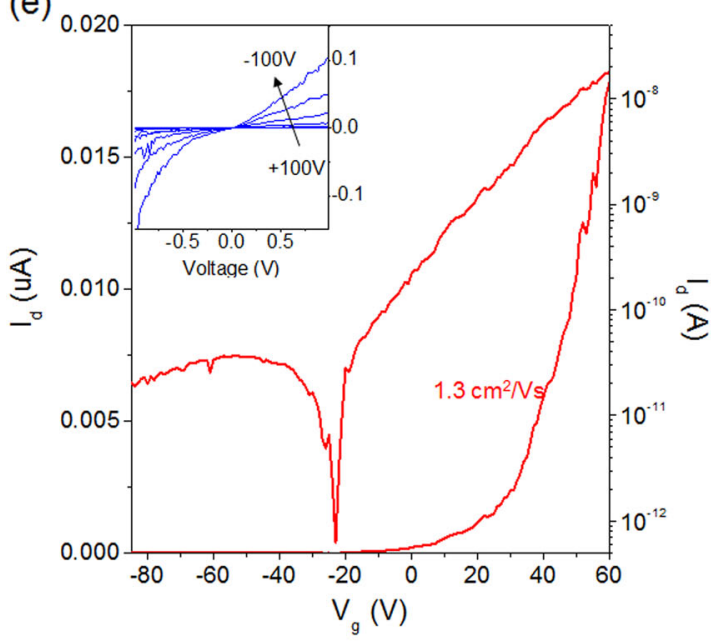

(c)

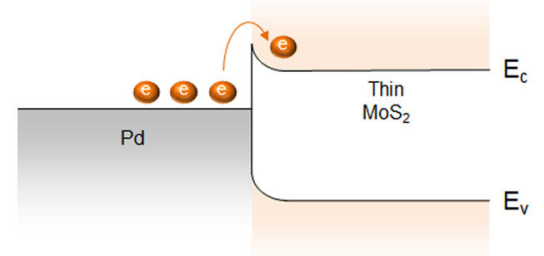

(d)

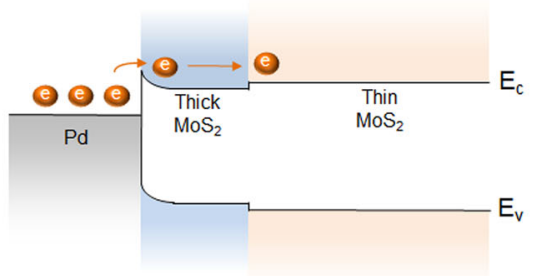

(f)

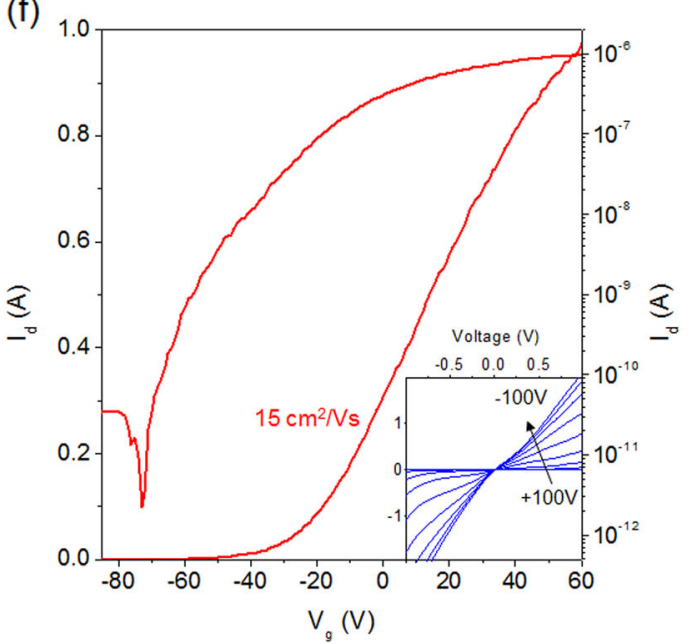

Fig. 6 a Schematic of FET devices with and without a thick $\mathrm{MoS}_{2}$ contact between thin $\mathrm{MoS}_{2}$ channel and Pd electrodes. b Optical image of FET device comprising two-layer (green dotted line) and six-layer (orange dotted line) $\mathrm{MoS}_{2}$. Energy-band diagrams for $\mathbf{c}$ thin $\mathrm{MoS}_{2} / \mathrm{metal}$ junction and

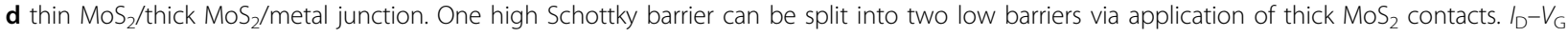
transfer curves for $\mathbf{e}$ device 1 and $\mathbf{f}$ device 2 (insets show corresponding $I_{D}-V_{D}$ output curves)

device, drain current shows a monotonous tendency without large temperature dependent change below $250 \mathrm{~K}$ and shows rectifying behavior like a Schottky junction due to the barrier at the junction (Fig. 5d). However, above $250 \mathrm{~K}$, the current level increases sharply and the rectifying ratio decreases. This is because the charge carrier was impeded by the junction barrier and the thermionic effect cannot appear under $250 \mathrm{~K}$. However, above $250 \mathrm{~K}$, the phonon-assist effect allows the charge carriers to sufficiently overcome the junction's barrier in NSJ. Conversely, in the LMJ, as the temperature increases, the drain current level initially increases before approximately $150 \mathrm{~K}$ and then decreases after approximately $200 \mathrm{~K}$, which is similar to an intrinsic $\mathrm{MoS}_{2}$ device (Fig. 5e). Since the barrier height of LMJ is relatively lower than that of NSJ, and the phonon-assist carrier appears before $77.4 \mathrm{~K}$, the level of drain current of the LMJ device is higher even at low temperature, and it shows more ohmic I-V characteristics than the NSJ device (Fig. 5c). These results are related to the phonon-assisted carrier and show good agreement with Fig. 4b. When the temperature is further increased, the drain current of LMJ decreases since the mobility decreases due to phonon scattering. Since the junction barrier height of LMJ is relatively small, the tendency of the drain current shows similar results as an intrinsic $\mathrm{MoS}_{2}$ device. Since an intrinsic $\mathrm{MoS}_{2}$ device does not have 
a junction other than the graphene contact, the overall trend is similar to LMJ devices (Fig. 5f). However, LMJ have small junction barriers such that the drain current decreases more when the temperature decreases.

As a result of the Arrhenius plot (Fig. S12), we calculated the barrier height. According to a previous study, and because multilayered graphene/ $\mathrm{MoS}_{2}$ heterostructure has negative Schottky barriers ${ }^{41}$, the results indicate that the junction barrier height at LMJ is much lower than the junction barrier height at NSJ, and this is enough for electrons to overcome the barrier by phonon-assist. Moreover, the barrier height difference between both $\mathrm{MoS}_{2}$ homojunctions is strong evidence that perfectly aligned band structure causes a stronger Fermi level pinning effect than a lattice-mismatched band structure.

\section{Contact resistance enhancement by lattice-mismatched homojunction effect}

A thicker $\mathrm{MoS}_{2}$ exhibits smaller contact resistance for high-work function metals owing to the lower Schottky barriers within them ${ }^{42}$. By utilizing the small junction resistance of the LMJ, we demonstrated high-performance $\mathrm{MoS}_{2}$ transistors with a reduced contact resistance leading to an enhanced two-terminal mobility. We fabricated two devices with different contact structures for comparison, as illustrated in Fig. 6a, b. When a drain bias was applied, thin $\mathrm{MoS}_{2}$ generally had a high Schottky barrier for high-work function metals leading to a large contact resistance and a reduced two-terminal field-effect mobility (Fig. 6c). However, thicker $\mathrm{MoS}_{2}$ had smaller Schottky-barrier heights owing to the lowered position of the conduction band. When thick $\mathrm{MoS}_{2}$ was inserted, charge carriers could be more easily transported into the metal (Fig. 6d). The output curves in Fig. 6e, $\mathrm{f}$ indicate that the $2 \mathrm{~L} \mathrm{MoS}_{2}$ device with Pd contacts had a high Schottky barrier. However, the $2 \mathrm{~L} \mathrm{MoS}_{2}$ device with the stacked contacts of $6 \mathrm{~L} \quad \mathrm{MoS}_{2}$ exhibited a dramatic improvement in the charge-carrier injection from the electrode to the channel.

In this study, we evaluated the junction decoupling effects of $\mathrm{MoS}_{2}$ layers for a lattice mismatched junction structure. SKPM and a junction-resistance evaluation suggested that the work function of $\mathrm{MoS}_{2}$ changed according to the number of layers and indicated the formation of a finite potential barrier at the layer-dependent $\mathrm{MoS}_{2}$ junction. Subsequent analysis revealed that the junction resistance for a lattice-mismatched state was far smaller than that for a perfectly aligned state. The small junction resistance of the LMJ structure was utilized for contact resistance reduction. The junction effects revealed may be useful in the development of new strategies that can be widely employed to engineer the energy-band structure in the heterostructure on the basis of the combination of various TMDs.

\section{Acknowledgements}

This research was partially supported by the Nanomaterial Technology Development Program (NRF-2017M3A7B4041987), Korean Government (MSIP) (No. 2015R1A5A1037668), and the National Research Foundation of Korea Grant funded by the Korean Government (Grant No.2017R1A5A1014862, SRC program: vdWMRC center).

\section{Author details}

${ }^{1}$ School of Mechanical Engineering, Yonsei University, Seoul 03722, Korea. ${ }^{2}$ Department of Materials Science and Engineering, Yonsei University, Seoul 03722, Korea. ${ }^{3}$ Dipartimento di Ingegneria dell'Informazione, Università di Pisa, Via G. Caruso, 16, 56122 Pisa, Italy. ${ }^{4}$ CNR-ICCOM, Consiglio Nazionale delle Ricerche, 56124 Pisa, Italy. ${ }^{5}$ Andlinger Center for Energy and the Environment, Princeton Univesity, Princeton, NJ 08544, USA. ${ }^{6}$ Department of Materials Science and Engineering, Seoul National University, Seoul 08826, Korea. ${ }^{7}$ Department of Electrical and Computer Engineering, University of Minnesota, Minneapolis, MN 55455, USA

\section{Authors contributions}

H.S.Y. and J.O. designed the experiment and wrote the manuscript. J.Y.P., J.K., and G.H.L. measured electronic characteristics and analyzed. J.K., T.L., and S.C.J. measured low temperature experiment and calculated Y-function, DFT and junction barriers. T.C., G.F., G.I., A.F., V.O.O. calculated DFT simulation.

Conflict of interest

The authors declare that they have no conflict of interest.

\section{Publisher's note}

Springer Nature remains neutral with regard to jurisdictional claims in published maps and institutional affiliations.

Supplementary information is available for this paper at https://doi.org/ 10.1038/s41427-019-0113-2.

Received: 17 May 2018 Revised: 15 September 2018 Accepted: 19 November 2018.

Published online: 5 April 2019

\section{References}

1. Pacile, D., Meyer, J., Girit, Ç. \& Zettl, A. The two-dimensional phase of boron nitride: few-atomic-layer sheets and suspended membranes. Appl. Phys. Lett. 92, 133107 (2008)

2. Lebegue, S. \& Eriksson, O. Electronic structure of two-dimensional crystals from ab initio theory. Phys. Rev. B 79, 115409 (2009).

3. Ling, $X$. et al. Raman enhancement effect on two-dimensional layered materials: graphene, h-BN and MoS2. Nano Lett. 14, 3033-3040 (2014).

4. Vogt, P. et al. Silicene: compelling experimental evidence for graphenelike two-dimensional silicon. Phys. Rev. Lett. 108, 155501 (2012).

5. Liu, C.-C., Feng, W. \& Yao, Y. Quantum spin Hall effect in silicene and twodimensional germanium. Phys. Rev. Lett. 107, 076802 (2011).

6. Radisavljevic, B., Radenovic, A., Brivio, J., Giacometti, I. V. \& Kis, A. Single-layer $\mathrm{MoS}_{2}$ transistors. Nat. Nanotechnol. 6, 147-150 (2011).

7. Li, L. et al. Black phosphorus field-effect transistors. Nat. Nanotechnol. 9 372-377 (2014).

8. Han, W.-Q., Wu, L., Zhu, Y., Watanabe, K. \& Taniguchi, T. Structure of chemically derived mono-and few-atomic-layer boron nitride sheets. Appl. Phys. Lett. 93, 223103 (2008).

9. Özçelik, V. O., Cahangirov, S. \& Ciraci, S. Stable single-layer honeycomblike structure of silica. Phys. Rev. Lett. 112, 246803 (2014).

10. Cahangirov, S., Topsakal, M., Aktürk, E., Şahin, H. \& Ciraci, S. Two-and onedimensional honeycomb structures of silicon and germanium. Phys. Rev. Lett. 102, 236804 (2009)

11. Das, S., Chen, H.Y., Penumatcha, A. V. \& Appenzeller, J. High performance multilayer $\mathrm{MoS}_{2}$ transistors with scandium contacts. Nano Lett. 13, 100-105 (2012). 
12. Lee, H. S. et al. $\mathrm{MoS}_{2}$ nanosheet phototransistors with thickness-modulated optical energy gap. Nano Lett. 12, 3695-3700 (2012).

13. Lin, Y.-C., Dumcenco, D. O., Huang, Y.-S. \& Suenaga, K. Atomic mechanism of the semiconducting-to-metallic phase transition in single-layered $\mathrm{MoS}_{2}$. Nat. Nanotechnol. 9, 391 (2014).

14. McDonnell, S., Addou, R., Buie, C., Wallace, R. M. \& Hinkle, C. L. Defectdominated doping and contact resistance in $\mathrm{MoS}_{2}$. ACS Nano 8, 2880-2888 (2014).

15. Zhang, Y., Ye, J., Matsuhashi, Y. \& Iwasa, Y. Ambipolar MoS 2 thin flake transistors. Nano Lett. 12, 1136-1140 (2012).

16. Bao, W., Cai, X., Kim, D., Sridhara, K. \& Fuhrer, M. S. High mobility ambipolar $\mathrm{MoS}_{2}$ field-effect transistors: substrate and dielectric effects. Appl. Phys. Lett. 102, 042104 (2013).

17. Deng, $\mathrm{Y}$. et al. Black phosphorus-monolayer $\mathrm{MoS}_{2}$ van der Waals heterojunction p-n diode. ACS Nano 8, 8292-8299 (2014).

18. Hong, X. et al. Ultrafast charge transfer in atomically thin $\mathrm{MoS}_{2}$ WS2 heterostructures. Nat. Nanotechnol. 9, 682 (2014).

19. Gong, Y. et al. Vertical and in-plane heterostructures from WS2/MoS 2 monolayers. Nat. Mater. 13, 1135 (2014)

20. Rivera, P. et al. Observation of long-lived interlayer excitons in monolayer MoSe2-WSe 2 heterostructures. Nat. Commun. 6, 6242 (2015).

21. Shi, Y. et al. van der Waals epitaxy of $\mathrm{MoS}_{2}$ layers using graphene as growth templates. Nano Lett. 12, 2784-2791 (2012).

22. Li, M.-Y. et al. Epitaxial growth of a monolayer $\mathrm{WSe}^{-}-\mathrm{MoS}_{2}$ lateral pn junction with an atomically sharp interface. Science 349, 524-528 (2015).

23. Preciado, E. et al. Scalable fabrication of a hybrid field-effect and acoustoelectric device by direct growth of monolayer $\mathrm{MoS}_{2} / \mathrm{LiNbO3}$. Nat. Commun. 6 , 8593 (2015).

24. Fu, L. et al. Direct growth of $\mathrm{MoS}_{2} / \mathrm{h}-\mathrm{BN}$ heterostructures via a sulfide-resistant alloy. ACS Nano 10, 2063-2070 (2016)

25. Chuang, S. et al. $\mathrm{MoS}_{2}$ p-type transistors and diodes enabled by high work function MoO x contacts. Nano Lett. 14, 1337-1342 (2014).

26. Jin, W. et al. Direct measurement of the thickness-dependent electronic band structure of $\mathrm{MoS}_{2}$ using angle-resolved photoemission spectroscopy. Phys. Rev. Lett. 111, 106801 (2013).

27. Han, S. et al. Band-gap transition induced by interlayer van der Waals interaction in MoS2. Phys. Rev. B 84, 045409 (2011).
28. Tosun, $\mathrm{M}$. et al. $\mathrm{MoS}_{2}$ heterojunctions by thickness modulation. Sci. Rep. $\mathbf{5}$ 10990 (2015).

29. Howell, S. L. et al. Investigation of band-offsets at monolayer-multilayer MoS junctions by scanning photocurrent microscopy. Nano Lett. 15, 2278-2284 (2015).

30. Ellis, J. K. Lucero, M. J. \& Scuseria, G. E. The indirect to direct band gap transition in multilayered $\mathrm{MoS}_{2}$ as predicted by screened hybrid density functional theory. Appl. Phys. Lett. 99, 261908 (2011)

31. Chhowalla, M. et al. The chemistry of two-dimensional layered transition metal dichalcogenide nanosheets. Nat. Chem. 5, 263-275 (2013).

32. Kuc, A., Zibouche, N. \& Heine, T. Influence of quantum confinement on the electronic structure of the transition metal sulfide T S 2. Phys. Rev. B 83, 245213 (2011).

33. Splendiani, A. et al. Emerging photoluminescence in monolayer $\mathrm{MoS}_{2}$. Nano Lett. 10, 1271-1275 (2010)

34. Ochedowski, O. et al. Effect of contaminations and surface preparation on the work function of single layer MoS 2 . Beilstein J. Nanotechnol. 5, 291 (2014).

35. He, J., Hummer, K. \& Franchini, C. Stacking effects on the electronic and optical properties of bilayer transition metal dichalcogenides $\mathrm{MoS}_{2}$, MoSe2, WS2, and WSe2. Phys. Rev. B 89, 075409 (2014).

36. Padilha, J., Peelaers, H., Janotti, A. \& Van de Walle, C. Nature and evolution of the band-edge states in $\mathrm{MoS}_{2}$ : from monolayer to bulk. Phys. Rev. B 90, 205420 (2014).

37. Li, T. \& Galli, G. Electronic properties of $\mathrm{MoS}_{2}$ nanoparticles. J. Phys. Chem. C. 111, 16192-16196 (2007).

38. Wang, K. et al. Interlayer coupling in twisted WSe2MS2 bilayer heterostructures revealed by optical spectroscopy. ACS Nano 10, 6612-6622 (2016).

39. Kim, K. et al. Raman spectroscopy study of rotated double-layer graphene: misorientation-angle dependence of electronic structure. Phys. Rev. Lett. 108, 246103 (2012).

40. Kaasbjerg, K., Thygesen, K. S. \& Jacobsen, K. W. Phonon-limited mobility in ntype single-layer $\mathrm{MoS}_{2}$ from first principles. Phys. Rev. B 85, 115317 (2012).

41. Qiu, D. \& Kim, E. K. Electrically tunable and negative Schottky barriers in multi-layered graphene/MoS 2 heterostructured transistors. Sci. Rep. 5, 13743 (2015).

42. Li, S.-L. et al. Thickness scaling effect on interfacial barrier and electrical contact to two-dimensional MoS, layers. ACS Nano 8, 12836-12842 (2014). 